\title{
INFLUÊNCIA DAS FONTES DE CARBONO E NITROGÊNIO NA PRODUÇÃO DE BIOSSURFACTANTE PELA RHODOTORULA LARYNGIS
}

\author{
H. A. CAMARGO $;$; T. G. C. SOUSA $;$; T. A. PINHEIRO 2 ; E. B. TAMBOURGI $;$ L. D. \\ $\mathrm{SETTE}^{4}$; A. PESSOA JR. $;$; V. L. CARDOSO ${ }^{2}$; U. COUTINHO-FILHO ${ }^{2}$ e E. SILVEIRA ${ }^{*} *$ \\ Universidade Estadual de Campinas, Faculdade de Engenharia Química. \\ ¿Universidade Federal de Uberlândia, Faculdade de Engenharia Química. \\ ${ }_{3}^{3}$ Universidade Federal de Uberlândia, Instituto de Genética e Bioquímica. \\ ${ }_{4}$ Universidade Estadual Paulista Campus Rio Claro, Departamento de Bioquímica e \\ Microbiologia. \\ sUniversidade de São Paulo, Faculdade de Ciências Farmacêuticas.E-mail para contato: \\ almeidacamargoster@gmail.com.br
}

\begin{abstract}
RESUMO - $\quad$ A produção de biosurfactantes, advém de microrganismos que são biodegradáveis, biocompatíveis e que possuem baixa toxicidade. Também observa-se que são estáveis em condições extremas como a variação do $\mathrm{pH}$, temperatura e salinidade. O presente trabalho teve como objetivo analisar a influência das fontes de carbono e nitrogênio na produção do biossurfactante pela levedura Rhodotorula laryngis, codificada como L69 e justifica-se devido ao seu interesse ambiental, industrial e farmacêutico. Para analises foram realizados índice de emulsificação em óleo mineral e teste de tensão superficial. Como resultado para as fontes de nitrogênio e carbono: resíduos de abacaxi, resíduos de caju, peptona, extrato de levedura e nitrato de amônia. Após essa seleção de melhores fontes de nitrogênio e carbono já é possível estabelecer planejamos para otimização da produção de biossurfactante, utilizando resíduos agroindustriais como fonte de carbono.
\end{abstract}

\section{INTRODUÇÃO}

Os biossurfactantes já são utilizados em diversos processos industriais, como na influência em processos de biorremediação (através do metabolismo dos microrganismos promover a diminuição ou erradicação de determinados poluentes em locais de extrema dificuldade), tornando os poluentes biodisponíveis a sua biodegradação; na agricultura agindo no controle biológico; na indústria de alimentos como substituto aos emulsificantes sintéticos em maioneses, molhos, sorvetes (BANAT et al.,2000) como também na área de cosméticos já que suas características umectantes o deixa mais compatível com a pele o que gera um menor grau de irritação. (MAIER e SOBERÓN -CHAVEZ, 2000)

Além disso, vale lembrar que os biossurfactantes advém de inúmeras possibilidades de formulações, as quais são principalmente influenciadas pelas fontes de carbono e de nitrogênio, entretanto a presença de fósforo, ferro, manganês e magnésio no meio de cultivo, podem maximizar a produção de biossurfactante. Outros pontos que potencializam o processo 
consiste na aferição do pH, temperatura, atmosfera, salinidade, agitação e condução de todo o processo. (BANAT, 1995).

Vale ressaltar que os biossurfactantes, apesarem de serem uma proposta biodegradável, ainda não são produzidos em grande escala para suportar todo o mercado industrial. Assim, visando o melhor custo benefício e praticidade de produção, a utilização de resíduos agroindustriais, como o abacaxi, no meio de cultura proporciona uma economia direta, já que a composição desses resíduos equivalem a 30\% dos biossurfactantes. Com isso o presente projeto possui como objetivo a determinar a melhor fonte de carbono e nitrogênio para a otimização do biossurfactante produzidos pela levedura Rodotorula laryngis nos meio de abacaxi e caju.

\section{MATERIAIS E METODOLOGIAS}

\subsection{Resíduos Agroindustriais}

Os resíduos agroindustriais de abacaxi (Ananas comosus) foram adquiridos nos mercados locais, onde o abacaxi in natura foi lavado com uma solução de hipoclorito de sódio a $10 \%$ (v/v) e processado no laboratório. Os resíduos constaram basicamente de de casca e folhas do abacaxizeiro e foram processados em moinho, já os resíduos agroindustriais de caju (Anacardium occidentale), passaram pelo mesmo processo e seus resíduos constaram basicamente do pedúnculo do fruto do cajueiro.

\subsection{Obtenção dos microrganismos}

A levedura da Antártica Rhodotorula laryngis, codificada por L 69, é proveniente da coleção de culturas da Divisão de Recursos Microbianos do Centro Pluridisciplinar de Pesquisas Químicas, Biológicas Agrícolas da Universidade Estadual de Campinas (CPQBA/Unicamp).

\subsection{Influência da fonte de Carbono e Nitrogênio na produção de biossurfactante}

Em Erlenmeyers de $200 \mathrm{ml}$ contendo meio YPD modificados para seleção de fonte de carbono e nitrogênio, foram inoculados o microrganismo. Utilizou-se como seleção para fonte de carbono: acetato de sódio, citrato de sódio, glicose, sacarose, peptona, extrato de levedura, extrato de abacaxi e extrato de caju, em quantidade suficiente para atingir a quantidade de $10 \mathrm{~g} / \mathrm{L}$, a qual é a mesma de açucares redutores do meio YPD. Já a fonte de nitrogênio foi realizada substituindo o extrato de levedura e peptona, pelos compostos: citrato de amônio, nitrato de amônio, sulfato de amônio, peptona e extrato de levedura, na concentração de $2 \mathrm{~g} / \mathrm{L}$. Por fim, para a produção dos meios foi necessário a utilização de uma solução salina com concentração de $10 \mathrm{~g} / \mathrm{L}, 5 \mathrm{~g} / \mathrm{L}, 2 \mathrm{~g} / \mathrm{L}$ e $0,2 \mathrm{~g} / \mathrm{L}$ de NaCl, Na2HPO4, KH2PO4 e MgSO4.7H2O, respectivamente, com fermentação de 56 horas a $15^{\circ} \mathrm{C}$.

\section{4 Índicie de Emulsificação (E24)}


Através da metodologia descrita por Cai et al. (2014) determinou-se o índice de emulsificação, que se baseia na mistura do óleo mineral com a solução aquosa livre de células do caldo fermentativo, na proporção de 1:1. Com o auxílio de um vortex, a mistura foi fortemente agitada e após 24 horas de repouso analisada. A taxa de emulsificação deu-se pela razão entre a altura da parte emulsificada e a altura total, e após convertida em porcentagem.

\subsection{Tensão Superficial}

Através do método do anel de du Noüy (1925), determinou-se a tensão superficial, a qual foi necessário a utilização do tensiômetro modelo K6 (Krüss GmbH, Humburgo, Alemanha) para medir a tensão superficial de $10 \mathrm{ml}$ de amostra da solução aquosa livre de células do caldo fermentativo à temperatura ambiente.

\section{RESULTADOS}

A figura 1 representa os resultados para o índice de emulsificação e de tensão superficial com o intuito de selecionar as melhores fontes de carbono e nitrogênio para a levedura L69.

Figura 1 - Tensão supeficial (mN.M-1) e índice de emulsificação em óleo mineral (\%), da levedura L 69 a. em diferentes fontes de nitrogênio; b. Em diferentes fontes de carbono. Legenda: Colunas pretas representam os resultados da tensão superficial e as colunas cinzas representam os dados do índice de emulsificação
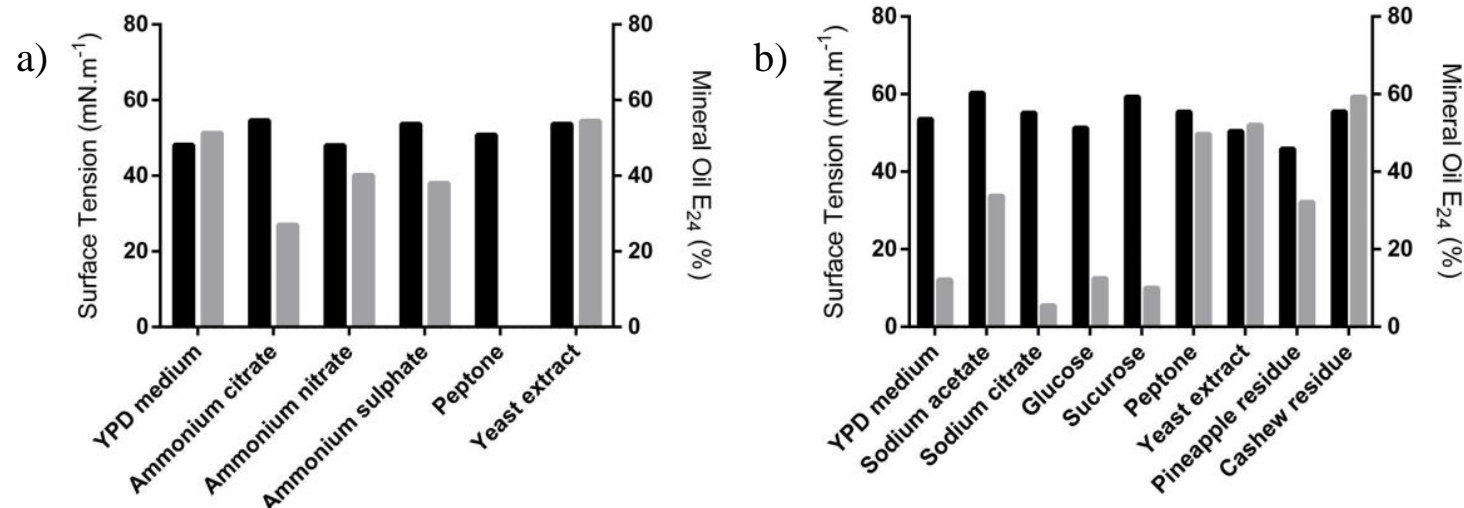

Conforme apresentado na figura 1a analisamos que os melhores resultados para o índice de emulsificação foram os que tiveram extrato de levedura e o nitrato de amônia como fonte de nitrogênio. Já para a tensão superficial destacaram o nitrato de amônia e peptona.

A figura $1 b$ demonstra os resultados para a seleção da fonte de carbono que se destacaram para o índice de emulsificação os compostos de extrato de levedura e resíduos de caju e para tensão superficial o melhor resultado obtido foram os resíduos de abacaxi e o extrato de levedura. 
A tensão superficial está ligada inversamente proporcional a produção de biossurfactante, enquanto o índice de emulsificação diretamente proporcional. Ambos geram dados que confirmam ou não a produção do biossurfactante. O índice de emulsificação consiste em um teste utilizado para confirmar a produção do biotensoativo no meio estudado, uma vez que já é conhecido que estas biomoléculas são capazes de modificar a hidrofobicidade da superfície celular além de promover a emulsão ou solubilização de soluções de óleo que antes seriam imiscíveis além disso tais biomoléculas são caracterizadas pela redução da tensão superficial. (Beal and Betts, 2000). Contudo há a possibilidade dessa biomolécula não ser produzida, mas mesmo assim apresente as demais propriedades, como a capacidade de formar emulsão. (YOUSSEF, et al., 2004).

Com os resultados obtidos, analisamos que as melhores fontes de carbono e nitrogênio que se enquadram em nosso estudo são: resíduos de abacaxi, resíduos de caju, peptona, extrato de levedura e nitrato de amônia.

\section{REFERÊNCIAS}

BANAT, I. M. Biosurfactant production and use in microbial enhanced oil recovery and possible uses in pollution remediation: A Review. Bioresource Technology, n.51, p.1-12, 1995.

BEAL, R.; BETTS, W. B. Role of rhamnolipid biosurfactants in the up take and mineralization of hexadecane in Pseudomonas aeruginosa. J. Appl. Microbiol., [s.1.], v. 89, p. 158-168, 2000.

CAI, Q., ZHANG, B., CHEN, B., ZHU, Z., LIN, W., CAO, T., 2014. Screening of biosurfactante producers from petroleum hydrocarbon contaminated sources in cold marine environments. Marine pollution bulletin, vol. 86, pp. 402-410.

DU NOÜY, P.L., 1925. An Interfacial Tensiometer for Universal Use. The Journal of General Physiology, vol. 7, pp. 625-633.

MAIER, R. M.; SOBERON-CHAVEZ, G. Pseudomonas aeruginosa rhamnolipids: biosynthesis and potential applications. Applied Microbiology and Biotechnology, [s.l.], v. 54, p. 625-633, 2000.

YOUSSEF, N. H.; DUNCAN, K. E.; NAGLE, D. P.; SAVAGE, K. N.; KNAPP, R. M.; MCLNERNEY, M. J. Comparasion of methods to detect biosurfactant production by diverse microrganism. Journal of Microbiological Methods, [s.1.], v. 56, p. 347-399, 2004. 